\title{
Diversified Pediatric Pharmacoepidemiology: An International Priority
}

\author{
Stuart M. MacLeod ${ }^{1}$
}

Published online: 23 August 2018

(c) Springer Nature Switzerland AG 2018

The article by Tomlin et al. [1] in this issue highlights an area that should be of critical concern for caregivers committed to optimizing pediatric drug therapy. Significant quality improvement in prescribing for children can only be achieved if all prescribers have reliable and comprehensive knowledge of current therapeutic choices. The New Zealand study is a useful demonstration of what can be learned from data available in a national pharmaceutical claims database capturing outpatient drug dispensing to all general practiceregistered children under 18 years of age between 2010 and 2015.

Studies such as this should encourage greater focus, in future, on prospective population-based investigations that will permit better understanding of choices among competing agents, both on and off label, and alternative formulations. The need for a broader vision of pharmacoepidemiology, one that embraces the concept of evidence-based medicine and its underlying scientific principles, is also implied.

While the retrieval of drug-dispensing data is commendable and of substantial value for future intercountry comparisons, it does have some important limitations. The approach taken, for instance, is likely to exclude a majority of critically ill children who receive most of their treatment exclusively in hospitals or other specialized centres. Any medications given parenterally will also be missed, as will products purchased over the counter. However, the latter omission may not be particularly important in New Zealand since it appears that, under the country's national insurance scheme, most medicines commonly sold over the counter elsewhere are covered by public funding and are consequently recorded in the national claims data. Understandably, such a policy

This comment refers to the article available at https://doi. org/10.1007/s40272-018-0303-3.

Stuart M. MacLeod

smacleod@bcchr.ca

1 Department of Pediatrics, University of British Columbia, Vancouver, BC, Canada decision in itself may significantly alter observed prescribing patterns in unpredictable ways.

The data presented give an accurate snapshot of outpatient prescribing and of changes occurring over a 5-year time span. Careful analysis of the demonstrated use patterns may offer insight into situations where particular products are undergoing extensive off-label use. Such observations should serve as a useful guide to needed clinical research and demand validation of efficacy for often routinely employed off-label treatments. In turn, the prevalence use data presented in Table 3 in Tomlin et al. [1] provoke a number of potential research questions, for example, possible overuse of medications such as ondansetron or underuse of treatments commonly prescribed in other jurisdictions for attention-deficit disorder with hyperactivity (ADDH). In the New Zealand data, only methylphenidate among candidate ADDH treatments appears and with a prevalence of use much lower than usually observed in other high-income countries [2]. The present report invitingly opens the door to legitimate queries concerning overzealous management of ADDH in the USA versus differing treatment standards in New Zealand. Careful evaluation of diagnostic criteria and critical comparison of therapeutic outcomes in both jurisdictions are called for.

Any interpretation of general drug claims data is likely to be complicated by the absence of a documented prescribing rationale, adherence indicators, and important details concerning dosage adjustment for age, weight and physical condition. Analysis is bound to be stymied, especially in any attempt at international comparisons, by the lack of universally agreed standards for treatment choice and dosage in prescribing for children. If this research field is to achieve all of its potential benefit, there must soon be an evolution to more closely targeted, hypothesis-driven, prospective pharmacoepidemiologic investigations that go beyond neutral reporting of dispensing data monitoring changes over time.

As an indirect conclusion, it should be noted that the scope of drug use studies must also be expanded to increasingly recognize the importance of epidemiologic research in low- and middle-income countries, where the major 
portion of the world's children reside. Particularly in poorly resourced settings, rational use of medicines is likely to be impeded by economic constraints as much as by regulatory policies, educational shortfalls, or practice irregularities [3]. Careful comparison between use patterns in low-income countries and high-income countries, such as New Zealand, may nonetheless help to direct prioritization of efforts to expand access to essential medicines and may foster the needed development of practical standardized treatment guidelines.

In 2017, my colleagues and I reported the results of an asset-mapping survey that attempted to identify a cross-section of physicians, pharmacists and researchers with a career focus on improving medicines use for children [4, 5]. Worldwide, there were almost 500 respondents to the survey, with a preponderance of individuals having professional qualifications in pharmacy or medicine. Approximately $8 \%$ of this highly specialized group reported clinical training in public or community health, and the same percentage, although often different individuals, described research interests in epidemiology related to prescribing and use of medications. It must be apparent that these numbers are shockingly inadequate to support the massive task ahead if pediatric pharmacotherapy is to be improved. It should also be noted that $87 \%$ of all survey respondents were working in high-income countries. This finding has disturbing implications for the prospects of better performance of much-needed pharmacoepidemiology research internationally.

Tomlin et al. [1] have provided an important example of the type of groundwork required if the ideal of rational use of medicines is going to be realized for children everywhere. Their study also serves to underscore the need for more finely detailed pediatric prescribing data and for an expanded emphasis on pharmacoepidemiology in all its dimensions by national governments, international agencies, academic institutions and philanthropic foundations addressing barriers to optimal child health posed by suboptimal use of medicines.

\section{Compliance with Ethical Standards}

Funding No sources of funding were used to prepare this manuscript.

Conflicts of Interest SM has no conflicts of interest that are directly relevant to the content of this article.

\section{References}

1. Tomlin AM, Woods DJ, Lloyd HS, Tilyard MW. Trends in outpatient prescription medicine use in New Zealand children 2010-2015: a national population-based study. Paediatr Drugs. 2018;20(5). https://doi.org/10.1007/s40272-018-0303-3.

2. Pennap D, Zito JM, Santosh PJ, et al. Patterns of early mental health diagnosis and medication treatment in a Medicaid-insured birth cohort. JAMA Pediatr. 2018;172(6):576-84. https://doi. org/10.1001/jamapediatrics.2018.0240.

3. Ranganathan SS, Gazarian M. Rational use of medicines (RUM) for children in the developing world: Current status, key challenges and potential solutions. In: MacLeod S, Hill S, Koren G, Rane A (Eds.). Optimizing treatment for children in the developing world. Switzerland: Springer International Publishing (Adis); 2015: p. 225-38.

4. MacLeod SM. Better therapies for children: A worldwide search for needed human resources. Paediatr Drugs. 2015;17(3):175-7.

5. MacLeod SM, Greff MJE, Knoppert DC, Ito S, Rieder MJ. An international asset map of clinicians, educators, and researchers pursuing better medicines use in children: initial findings. Clin Pharmacol Ther. 2017;101:274-80. 